\title{
Evaluation of Trochlear Dysplasia in Dogs with Medial Patellar Luxation - Comparative Studies
}

\author{
Radka Stayova Garnoeva
}

\begin{abstract}
Background: Medial patellar luxation (MPL) is one of the commonest orthopaedic diseases in small dog breeds. Although the bone deformities associated with canine medial patellar luxation are described in numerous studies, the pathogenesis of the condition is still disputable. What is more, there is no categorical evidence that luxation of the patella is associated to a shallow trochlear groove as no objective method for determination of trochlear depth and shape has been proposed. The aim of the present study was to evaluate the depth and shape of femoral trochlear groove on radiographs obtained from healthy dogs and dogs affected with grade II and grade III MPL.

Materials, Methods \& Results: A total of 45 dogs (33 with MPL and 12 healthy) from 4 small breeds (Mini-Pinscher, Pomeranian, Chihuahua and Yorkshire terrier) were included in the study. After deep sedation, stifle radiographs were obtained in tangential projection (skyline view). The dogs were positioned in ventral recumbency, the examined stifle bent as much as possible, and the central beam focused on the patella between femoral condyles. Six morphometric parameters associated with the onset of trochlear dysplasia similar to those used in human medicine were measured: trochlear sulcus angle (SA), lateral and medial trochlear inclination angles (LTI; MTI), trochlear groove depth (TD), patellar thickness (PaT) and the ratio between trochlear depth and patellar thickness (PaT/TD). The non-parametric Mann-Whitney test was used for evaluation of differences between healthy joints and those affected with grade II and III MPL. The association between measured variables was evaluated via the Spearman's rank-order correlation. TD was greater in healthy joints as compared to those affected with MPL grade II and III $(P<0.001)$. In healthy stifles, PaT value exceeded significantly $(P$ $<0.01)$ that in joints with grade III MPL. The TD/PaT ratio was significantly greater in healthy joints vs both those with grade II $(P<0.01)$ and grade III MPL $(P<0.001)$. In healthy joints, there was a significant negative relationship (rho $-0.508 ; P=0.0113$ ) between SA and TD: smaller sulcus angles corresponded to deeper trochleas. This correlation was even stronger in joints with patellar luxation (rho $-0.723 ; P<0.0001$ ). The LTI and MTI showed a very strong positive correlation in healthy joints (rho $0.854 ; P<0.0001$ ) and at the same time, lack of significant association in joints affected with MPL (rho 0.163; $P=0.327$ for grade II MPL and rho $0.175 ; P=0.448$ for grade III MPL) was demonstrated. The altered trochlear shape and depth were more pronounced in joints with grade III MPL. As MPL grade increased, the SA became statistically significantly greater. In grade III MPL it was accompanied with considerably reduced trochlear depth, medial trochlear inclination angle and trochlear depth/patellar thickness ratio.

Discussion: Five of the measured morphometric parameters for radiographic detection of trochlear dysplasia in dogs were found to be important in the evaluation of trochlear morphology in dogs. The obtained results indicated the presence of trochlear dysplasia in dogs with MPL. A 3-stage classification system for assessment of abnormal trochlear development in small dog breeds: mild; moderate and severe trochlear dysplasia, was proposed. The occurrence of shallow trochlear groove and medial femoral condyle's hypoplasia could be accepted as signs of mild and moderate trochlear dysplasia. The pre-operative measurements of these parameters could improve surgical planning and decisions-making.
\end{abstract}

Keywords: medial patellar luxation, trochlear dysplasia, trochlear depth, small dog breeds. 


\section{INTRODUCTION}

The trochlear groove is formed by lateral and medial condyles and a well-shaped groove between (sulcus patellaris) [28]. Sometimes, the groove could be abnormally shaped (shallow, flat or dome-shaped): this alteration is referred to as trochlear dysplasia [7,23].

In men, trochlear dysplasia (TD) was first described by the French surgeon Richerand, who reported several cases of patellar luxation with shorter medial femoral condyle. TD was first classified [12] as moderate (shallow trochlea, normal shape) and severe (shallow trochlea, no visible sulcus). Later, the classification was modified to 4 types [8]: A- shallow normally-shaped trochlea; B- flat trochlea; C- hypoplasia of the medial condyle, trochlear asymmetry; D- convex trochlea. The shallow trochlea decreases patellofemoral joint congruity, resulting in patellar instability and luxation [6].

Medial patellar luxation (MPL) is one of the commonest orthopaedic diseases in small dog breeds $[1,3]$. Although the bone deformities associated with MPL are well described, its pathogenesis is disputable [13]. In veterinary medicine, no criteria related to trochlear shape and depth are adopted as there is no evidence that patellar luxation is due to shallow trochlear groove [27]. Also, no objective method for determination of trochlear depth has been proposed [26]. The present study aimed to evaluate some morphometric parameters of femoral trochlear groove on tangential radiographs from healthy dogs and dogs with grade II and III MPL to determine trochlear shape and depth parameters, associated with this condition.

\section{MATERIALS AND METHODS}

\section{Study cohort}

The study was performed with 45 dogs from 4 small breeds (18 Mini-Pinschers; 13 Pomeranians; 9 Chihuahuas and 5 Yorkshire terriers). Of them, 33 dogs (53 stifle joints) were diagnosed with medial patellar luxation (MPL) - 31 joints with MPL grade II and 21 joints with MPL grade III after physical examination and radiography. The grade of MPL was determined by the routine clinical classification [21]. The other 12 dogs from the same breeds ( 24 joints) had no orthopaedic or neurological affections and were used as controls.

\section{Radiography and measurements}

After deep sedation with $0.075 \mathrm{mg} / \mathrm{kg}$ medetomidine hydrochloride ${ }^{1}$ [Dorbene vet $^{\circledR}-1$ $\mathrm{mg} / \mathrm{mL}]$ and $7.5 \mathrm{mg} / \mathrm{kg}$ ketamine hydrochloride ${ }^{2}$ [Anaket ${ }^{\circledR}-100 \mathrm{mg} / \mathrm{mL}$ ] applied together into $m$. quadriceps femoris, radiographs were obtained in tangential projection (skyline view) using a Bucky Diagnost CS4 stationary X-ray equipment ${ }^{3}$ with iQ-CR ACE acquisition station and iQ-VIEW/PRO version 2.7. software (exposure data $50 \mathrm{kV}, 10 \mathrm{mAs}$ ). The dogs were positioned in ventral recumbency, the examined stifle bent as much as possible, and the central beam focused on the patella between femoral condyles.

All trochlear angles, trochlear depth and patellar thickness were measured on tangential radiographs. The sulcus angle (Figure 1) is formed by lines connecting each of lateral and medial condyles with the deepest point of the trochlea [4]. The lateral trochlear inclination (LTI) is defined as the angle formed by the line tangential to the posterior condyle and the line passing from the central sulcus to the lateral condyle [14] whereas the medial trochlear inclination angle (MTI) is formed by the line tangential to the posterior condyle and the line passing from the central sulcus to the medial condyle (Figure 1).

For measurement of the trochlear depth (TD), a line was passed through the lateral and medial trochlear facets, and a perpendicular line was drawn from the trochlear groove bottom [20]. The segment between the point of interception of above described lines and the deepest part of trochlear groove is defined as TD (Figure 2). The patellar thickness (PaT) was also measured [25].

\section{Statistical analysis}

Non-parametric Mann-Whitney test was used for evaluation of differences between healthy joints and those affected with MPL at the $P<0.05$ level. The association between measured variables was evaluated via the Spearman's rank-order correlation. The tests were performed by statistical software (MedCalc v. 10.2 .0 .0$)^{4}$. 


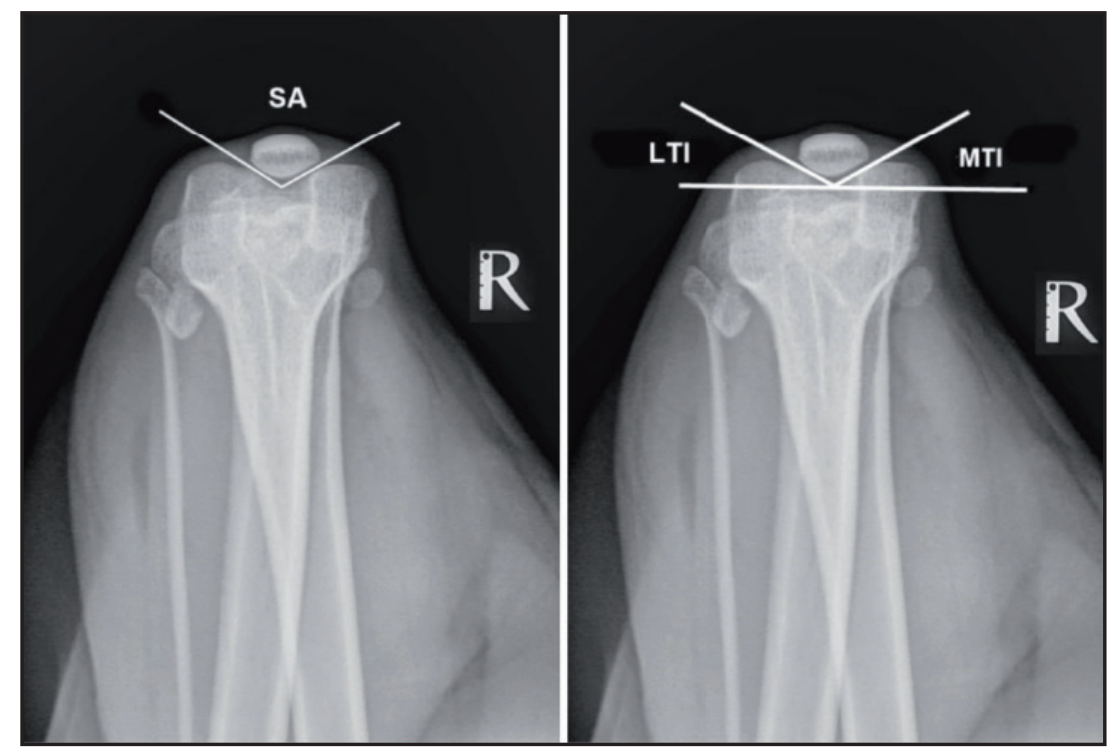

Figure 1. Left - Measurement of trochlear sulcus angle (SA) [4]. Right - measurement of lateral and medial trochlear inclination (LTI; MTI) [14].

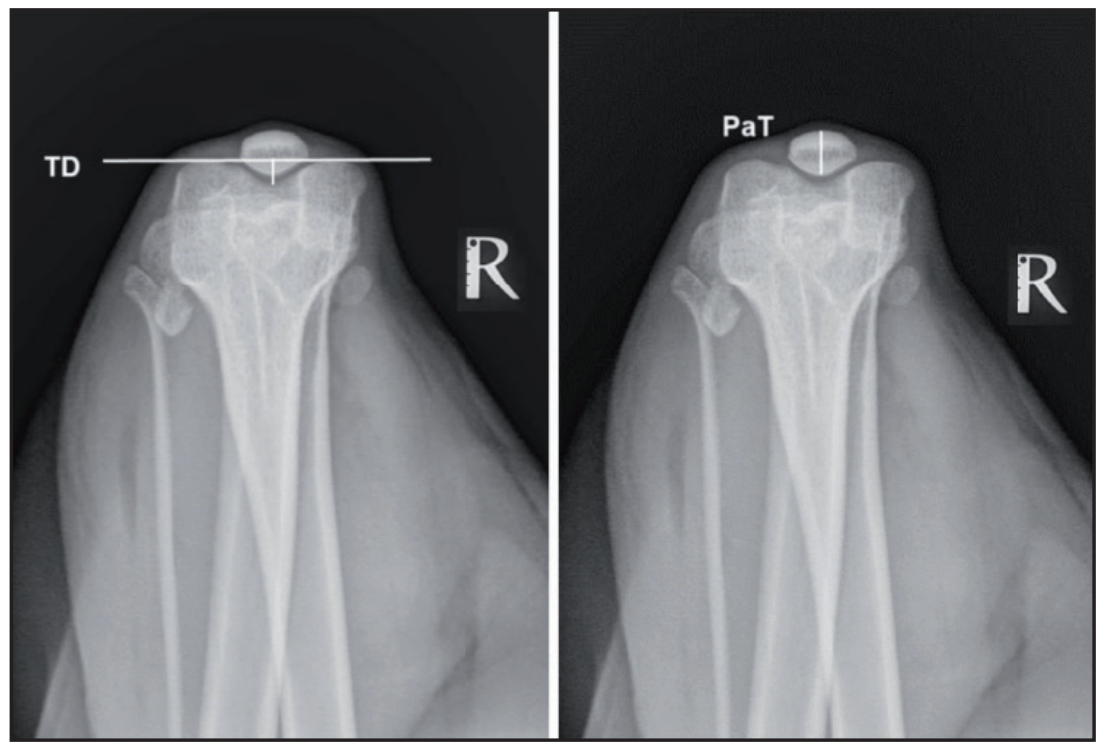

Figure 2. Left - Measurement of trochlear depth (TD) [20]. Right - measurement of patellar thickness $(\mathrm{PaT})$ [25].

\section{RESULTS}

The results from measured parameters in healthy dogs and dogs affected by MPL grade II and III from the 4 studied breeds (Mini-Pinscher, Pomeranian, Chihuahua, Yorkshire terrier) are presented in Table 1.

The sulcus angle (SA) differed statistically significantly between healthy joints and joints with grade II and III MPL. It was smaller in healthy joints $\left(125^{\circ}\right)$ vs joints with MPL grade II $\left(134^{\circ}\right)[P<0.005]$ and
MPL grade III $\left(145^{\circ}\right)[P<0.0001]$ (Table 1$)$. The SA values were also significantly different between both studied grades of medial patellar luxation $(P<0.0001)$. Lateral and medial trochlear inclination angles (LTI; MTI) were substantially $(P<0.0001)$ smaller in stifle joints with grade III MPL (LTI - 23; MTI - 19 ${ }^{\circ}$ ) compared to healthy joints (LTI $-28.5^{\circ}$; MTI $-27.5^{\circ}$ ). A similar tendency, yet with statistically insignificant differences was demonstrated for joints with grade II MPL (Table 1). 
It should be noted that trochlear depth (TD) was greater in healthy joints $(2.05 \mathrm{~mm})$ as compared to those affected with MPL grade II (1.2 $\mathrm{mm})$ and MPL grade III $(0.8 \mathrm{~mm})[P<0.001]$

In normal stifle joints, the patellar thickness (PaT) exceeded significantly $(P<0.01)$ that in joints with grade III MPL $(3.15 \mathrm{~mm}$ and $2.7 \mathrm{~mm}$, respectively). PaT in joints with grade II MPL had intermediate values, but differences with the other groups were insignificant. As the TD/PaT ratio was concerned, it was significantly greater in healthy joints $(0.65)$ vs both those with grade II MPL $(0.46 ; P<0.01)$ and grade III MPL $(0.33 ; P<0.001)$. It was found out that this ratio differed statistically significantly between joints with grade II and III MPL $(0.46$ and $0.33 ; P<$ 0.01) [Table 1].

In healthy joints, there was a significant negative relationship (rho $-0.508 ; P=0.0113$ ) between SA and TD: smaller sulcus angles corresponded to deeper trochleas. In joints with grade II and III MPL, this correlation was even stronger (rho $-0.723 ; P<0.0001$ ). Another interesting relationship was found out between LTI and MTI in the different groups of joints - a very strong positive correlation of these 2 angles in healthy joints (rho 0.854; $P<0.0001$ ) and at the same time, lack of significant association in joints affected with MPL (rho $0.163 ; P=0.327$ for grade II MPL and rho $0.175 ; P=0.448$ for grade III MPL).

Table 1. Trochlear angles, trochlear depth and patellar thickness in healthy canine stifle joints and joints with medial patellar luxation (MPL) grade II and III. Data are presented as median (minimum-maximum).

\begin{tabular}{|c|c|c|c|c|}
\hline & $\begin{array}{l}\text { Healthy joints } \\
\qquad(\mathrm{n}=24)\end{array}$ & $\begin{array}{l}\text { MPL grade II joints } \\
\qquad(\mathrm{n}=32)\end{array}$ & $\begin{array}{l}\text { MPL grade III joints } \\
\qquad(\mathrm{n}=21)\end{array}$ & Level of significance \\
\hline & $\mathrm{j}$ & $\mathrm{k}$ & 1 & \\
\hline $\mathrm{SA},{ }^{\circ}$ & $125(112-153)$ & $134(126-138)$ & $145(135-160)$ & $\begin{array}{c}P_{1-2}<0.05 \\
P_{1-3}<0.001 \\
P_{2-3}<0.001\end{array}$ \\
\hline LTI, $^{\circ}$ & $28.5(12-39)$ & $26.5(23-35)$ & $23(11-30)$ & $\begin{array}{c}P_{1-3}<0.001 \\
P_{2-3}<0.01\end{array}$ \\
\hline MTI, ${ }^{\circ}$ & $27.5(14-35)$ & $26.5(19-32)$ & $19(9-28)$ & $\begin{array}{l}P_{1-3}<0.001 \\
P_{2-3}<0.001\end{array}$ \\
\hline $\mathrm{TD}, \mathrm{mm}$ & $2.05(0.7-3.1)$ & $1.2(0.9-1.9)$ & $0.8(0.5-1.2)$ & $\begin{array}{l}P_{1-2}<0.001 \\
P_{1-3}<0.001 \\
P_{2-3}<0.001\end{array}$ \\
\hline PaT, mm & $3.15(1.8-5.0)$ & $2.9(1.9-4.1)$ & $2.7(1.5-3.5)$ & $P_{1-3}<0.01$ \\
\hline $\mathrm{TD} / \mathrm{PaT}$ & $0.65(0.35-1.00)$ & $0.46(0.25-0.71)$ & $0.33(0.17-0.48)$ & $\begin{array}{c}P_{1-2}<0.01 \\
P_{1-3}<0.001 \\
P_{2-3}<0.01\end{array}$ \\
\hline
\end{tabular}

n- number of joints; SA- trochlear sulcus angle; LTI- lateral trochlear inclination; MTI- medial trochlear inclination; TA- trochlear angle; TD- trochlear depth; PaT- patellar thickness.

\section{DISCUSSION}

Trochlear dysplasia is a condition characterised with abnormally developed trochlear groove $[4,7,8,20,23]$. It is described in detail in humans and its radiographic appearance was classified into 4 types: $\mathrm{A} ; \mathrm{B} ; \mathrm{C}$ and D [7,8], yet its existence in animals was not confirmed so far. Despite the numerous apparently identical morphometric parameters proposed by re- searchers, the different methods for their measurement greatly influence the reported values [27]. In this study, our attention was focused on sulcus angle, lateral and medial inclination angles, trochlear depth and patellar thickness. In our belief, when measured on tangential radiographs, these parameters allowed a satisfactory evaluation of trochlear groove and patella. Each change in trochlear groove shape results in altered biomechanics of patellofemoral joints and thus, instability $[5,19]$. 
The proper function of this joint requires alignment of m. quadriceps femoris, the patella, trochlear groove, the straight patellar ligament and crista tibiae $[9,10]$. Deviation of one or more structures from this line leads to altered direction and reduced strength of the entire quadriceps mechanism [22].

In human medicine, trochlear morphometry is performed in lateral and axial views [4,7]. Axial views provide a more accurate trochlear groove assessment $[18,20]$. In the present study, the tangential (skyline) view with completely flexed stifle joint was used due to the better visualization of the trochlea. This projection allowed better evaluation of trochlear depth, patellar thickness and trochlear condyles' asymmetry as dogs.

It is affirmed that patellar luxation occurs consequently to complex morphological musculoskeletal abnormalities affecting the entire pelvic limb, with leading pathogenetic role of the abnormal trochlear groove development e.g. dysplasia [3,16,29]. So far, the incidence of trochlear dysplasia in dogs has not been reported [27]. The author states that true trochlear dysplasia was very rarely encountered and that most cases referred only to shallow trochlear groove due to hypoplasia of the medial femoral condyle. In our opinion, even the presence of a shallow trochlea in dogs could be defined as a mild degree of trochlear dysplasia, because trochlear condyles remained short, and trochlear depth and shape are determined by their height. The shallow trochlea in men, according to the Dejour's classification [8] is termed trochlear dysplasia type A.

In available scientific literature, only 2 studies reported the trochlear depth and shape in healthy dogs but in medium-size and large breeds $[15,17]$ although medial patellar luxation is commonly seen in small dog breeds. That is why our attention was focused on 4 small breeds: Mini-Pinscher, Pomeranian, Chihuahua and Yorkshire terrier.

One of the most important trochlear angles is the sulcus angle. In healthy human stifle joints, SA is $135^{\circ}$ on the average - when SA becomes $145^{\circ}$ or greater, the trochlea is defined as shallow and is considered prerequisite for occurrence of patellar luxation [20]. In the present study in dogs, SA in healthy joints was $125^{\circ}$, and it increased in joints affected with MPL ( $134^{\circ}$ in grade II and $145^{\circ}$ in grade III). This was due to the smaller height of trochlear facets (the medial one in particular) in joints diagnosed with medial patellar luxation. The smaller height of trochlear facets was associated with higher sulcus angle and shallower groove - confirmed by values of lateral and medial inclination angles (LTI and MTI). These are the second important trochlear angles indicating femoral condylar asymmetry on radiographs [14]. In studied dogs, the medial inclination angle tended to be smaller in joints affected with patellar luxation compared to healthy joints. What is more, MTI was smaller than LTI in joints with grade III MPL. Possibly, this was due to progressive reduction of medial trochlear facet height as the patella exerted a stronger pressure on medial bone and soft tissue structures.

This may explain the predisposition of small dog breeds to MPL, yet more studies are necessary to support this hypothesis. Human studies have established that medial femoral condyle hypoplasia is a primary factor for onset of trochlear dysplasia and patellofemoral instability [11]. In the present study, hypoplasia of the medial condyle was also confirmed by smaller values of the MTI in relation to LTI, but only in dogs affected by grade III luxation - a finding corresponding to TD Dejour type C [8].

Up to $50 \%$ of the patella should fit into the normally developed trochlear groove [24]. Dogs with MPL have abnormal trochlear groove varying from almost normal to absent trochlear groove [9]. The present study has shown that TD of joints with grade II and III MPL was statistically significantly smaller than that of healthy joints $(P<0.001)$, and less than $50 \%$ of patellae were positioned within the groove, confirmed by data about ratio of TD and patellar thickness. This ratio was greater in healthy joints in which trochlear depth and shape fits ideally the patellar thickness. On the contrary, in diseased joints, the TD/PaT ratio was greatly reduced especially in grade III MPL. The shallow trochlear alters the extensor mechanism and disturbs the normal articulation of patella in the trochlear groove.

The trochlear depth in dogs is breed-dependent. A research team [15] reported shallower trochlea in healthy French and English bulldogs as compared to Pug dogs. Other authors used conventional radiography, ultrasonography and stereomicroscopy for measurement of trochlear groove depth in small dog cadavers (Pintchers, Chihuahua, Shih-Tzu, mixed breeds up to $7.5 \mathrm{~kg}$ weight) without signs of orthopaedic disease and concluded that TD values measured on radiographs were the highest (TD - $1.31 \mathrm{~mm}$ ) [2]. TD values in our study were higher (TD - $2.05 \mathrm{~mm}$ ) probably due to the used 
tangential radiographic view, which could be hardly performed in cadaveric studies as it is impossible to obtain a fully flexed stifle due to rigor mortis.

Five out of the measured 6 morphometric parameters adopted from human medicine for radiographic detection of trochlear dysplasia in dogs were found to be important in the evaluation of trochlear morphology in dogs. The obtained results indicated the presence of trochlear dysplasia in dogs. In our study, a 3-stage classification system for assessment of abnormal trochlear development in small dog breeds is proposed: mild; moderate and severe trochlear dysplasia. Thus, in mild TD, trochlear groove is shallow, with symmetric femoral condyles, trochlear depth < $2.0 \mathrm{~mm}$, sulcus angle $>126^{\circ}$, and trochlear depth/patellar thickness ratio $(\mathrm{TD} / \mathrm{PaT})<0.5(50 \%)$. In stifles with moderate TD, femoral condyles are asymmetrical (hypoplasia of the medial condyle), medial trochlear inclination of $20^{\circ}$ or less, trochlear depth $<1.0 \mathrm{~mm}$, sulcus angle $145^{\circ}$ or greater, and TD/PaT: $33 \%$ or lower. In all other instances characterised with greater deviations of above mentioned morphometric parameters, the trochlear dysplasia could be defined as severe.

\section{CONCLUSION}

In conclusion, tangential radiographs of canine stifle joints allowed a satisfactory evaluation of trochlear groove depth and shape. Increased sulcus angle values along with substantially lower trochlear depth, medial trochlear inclination and smaller patellar thickness to trochlear depth ratio suggested altered trochlear shape and depth.

Trochlear dysplasia may be an important element in the etiopathogenesis of medial patellar luxation in small dog breeds. The shallow trochlear groove and medial femoral condyle's hypoplasia could be accepted as signs of mild and moderate trochlear dysplasia. The pre-operative measurements of these parameters could improve surgical planning and decisions-making.

\author{
MANUFACTURERS \\ ${ }^{1}$ Laboratorios Syva S.A. León, Spain. \\ ${ }^{2}$ Richter Pharma. Wels, Austria. \\ ${ }^{3}$ Philips Bucky Diagnost. Amsterdam, Netherlands. \\ ${ }^{4}$ MedCalc Software. Oostende, Belgium.
}

Declaration of interest. The author reports no conflicts of interest and is responsible for the content and writing of the paper.

\section{REFERENCES}

1 Alam M.R., Lee J.I., Kang H.S., Kim I.S., Park S.Y. \& Lee K.C. 2007. Frequency and distribution of patellar luxation in dogs. 134 cases (2000 to 2005). Veterinary Comparative Orthopaedics and Traumatology. 20: 59-64.

2 Boonchaikitanan P., Choisunirachon N. \& Soontornvipart K. 2019. A feasibility of ultrasonographic assessment for femoral trochlear depth and articular cartilage thickness in canine cadavers. Thai Journal of Veterinary Medicine. 49(3): 257-264.

3 Bound N., Zakai D., Butterworth S.J. \& Pead M. 2009. The prevalence of canine patellar luxation in three centres. Clinical features and radiographic evidence of limb deviation. Veterinary Comparative Orthopaedics and Traumatology. 22(1): 32-37.

4 Brattström H. 1964. Shape of the intercondylar groove normally and in recurrent dislocation of patella: a clinical and X-ray anatomical investigation. Acta Orthopaedica Scandinavica. 68 (suppl): 1-148.

5 Carneiro R.K., Souza M.J., Bing R.S., Alievi M.M., Feliciano M.A. \& Ferreira M.P. 2020. Radiographic assessment of the depth of the trochlear groove and patellar diameter in dogs. Acta Scientiae Veterinariae. 48: 1754. 7p.

6 Davies A.P., Costa M.L., Shepstone L., Glasgow M.M. \& Donell S. 2000. The sulcus angle and malalignment of the extensor mechanism of the knee. Journal of Bone and Joint Surgery (British volume). 82(8): 1162-1166.

7 Dejour H., Walch G., Neyret P. \& Adeleine P. 1990. La dysplasie de la trochlée fémorale. Revue de Chirurgie Orthopédique et Réparatrice de l'Appareil Moteur. 76(1): 45-54.

8 Dejour D., Reynaud P. \& Lecoultre B. 1998. Douleurs et instabilité rotulienne, Essai de classification. Médecine et Hygiène. 56(2217): 1466-1471.

9 Hulse D.A. 1981. Pathophysiology and management of medial patellar luxation in the dog. Veterinary Medicine: Small Animal Clinician. 76: 43-51.

10 Hulse D.A. 1995. The Stifle Joint. In: Olmstead M.L. (Ed). Small Animal Orthopedics. St. Louis: Mosby, pp.395-404.

11 Keshmiri A., Schöttle P. \& Peter C. 2020. Trochlear dysplasia relates to medial femoral condyle hypoplasia: an MRIbased study. Archives of Orthopaedic and Trauma Surgery. 140: 155-160. 
12 Knutsson F. 1941. Uber die rontgenologie des femoropatellargelenks sowie eine gute projection fur das kniegelenk. Acta Radiologica. 22: 371-376.

13 Kowaleski M.P., Boudrieau R. \& Pozzi A. 2012. Stifle joint. In: Tobias K. \& Johnston S. (Eds). Veterinary Surgery: Small Animal. St. Louis: Saunders Elsevier, pp.973-979.

14 Laurin C.A., Lévesque H.P., Dussault R., Labelle H. \& Peides J.P. 1978. The abnormal lateral patellofemoral angle: a diagnostic roentgenographic sign of recurrent patellar subluxation. Journal of Bone and Joint Surgery. 60(1): 55-60.

15 Matchwick A., Bridges J.P., Mielke B., Pead M.J., Phillips A. \& Meeson R.L. 2021. Computed tomographic measurement of trochlear depth in three breeds of brachycephalic dogs. Veterinary Comparative Orthopaedics and Traumatology. 34(2): 124-129.

16 Mortari A.C., Rahal S.C., Vulcano L.C., Silva V.C. \& Volpi R.S. 2009. Use of radiographic measurements in the evaluation of dogs with medial patellar luxation. Canadian Veterinary Journal. 50: 1064-1068.

17 Nicetto T., Longo F., Contiero B., Isola M. \& Petazzoni M. 2020. Computed tomographic localization of the deepest portion of the femoral trochlear groove in healthy dogs. Veterinary Surgery. 49(6): 1246-1254.

18 Pace J.L., Cheng C., Sheeba M., Joseph M.D. \& Solomito M.J. 2020. Effect of trochlear dysplasia on commonly used radiographic parameters to assess patellar instability. The Orthopaedic Journal of Sports Medicine. 8(7): doi: $10.1177 / 2325967120938760$.

19 Petazzoni M., Troiano D., Denti F., Buiatti M. \& De Giacinto E. 2018. Computed Tomographic Trochlear Depth Measurement in Normal Dogs. Veterinary and Comparative Orthopaedics and Traumatology. 31(6): 431-437.

20 Pfirrmann C.W., Zanetti M., Romero J. \& Hodler J. 2000. Femoral trochlear dysplasia: MR findings. Radiology. 216(3): 858-864.

21 Putnam R.W. 1968. Patellar Luxation in the Dog. 111p. Guelph, Canada. Master of Science. University of Guelph.

22 Schulz K.S. 2007. Diseases of the joints. In: Fossum T.W. (Ed). Small Animal Surgery. 3rd edn. St Louis: Mosby, pp.1289-1297.

23 Sharma N., Brown A., Bouras T., Kuiper J. H., Eldridge J. \& Barnett A. 2020. The Oswestry-Bristol classification: a new classification system for trochlear dysplasia. Bone and Joint Journal. 102-B(1): 102-107.

24 Slocum B. \& Slocum T.D. 1993. Trochlear wedge recession for medial patellar luxation. Veterinary Clinics of North America: Small Animal Practice. 23(4): 869-875.

25 Stäubli H.U., Dürrenmatt U., Porcellini B. \& Rauschning W. 1999. Anatomy and surface geometry of the patellofemoral joint in the axial plane. Journal of Bone and Joint Surgery. 81: 452-428.

26 Talcott K.W., Goring R.L. \& De Hann J.J. 2000. Rectangular recession trochleoplasty for treatment of patellar luxation in dogs and cats. Veterinary Comparative Orthopaedics and Traumatology. 13: 39-43.

27 Van der Zee J. 2010. The cranial sartorius muscle, peculiar strap like strand, or prominent muscle and significant contributor to MPL? In: Proceedings of the 15th Congress of the European Society of Veterinary Orthopaedics and Traumatology (Bologna, Italy). p.694.

28 Vasseur P. B. 2003. Stifle joint. In: Slatter D. (Ed). Textbook of Small Animal Surgery. 3rd edn. Philadelphia: Saunders, pp.2090-2133.

29 Wangdee C. \& Torwattanachai P. 2010. Lateral patellar luxation in three Pomeranian dogs: A case report. Thai Journal of Veterinary Medicine. 40(2): 227-231. 